\title{
Lumbar shape factors extraction influencing the ratio of waist- darts' volumes for young female
}

\author{
Xue Zhang, Hongshu Jin \\ Wuhan Textile University, Wuhan, Hubei, 430073, China
}

\begin{abstract}
To improve the fitness of bottoms of individuals, this study is proposed to extract lumbar shape factors influencing the ratio of waist dart's volumes of young females. A total of 27 measurement items including lumbar shapes and waist dart's volumes were collected through overlapped graphics of lumbar feature sections obtained from 30 female college students aged 18 and 24via 3D body scanning method. Results of correlation analysis showed that the total waist dart's volume was significantly correlated with the lateral lumbar bending level (ytc), and the ratio of each waist dart's volume was significantly correlated with lumbar anteversion angle $(\beta)$, lumbar anteverted amount (ytq) and buttock projected volume $(\mathrm{ab})$ respectively with a correlation coefficient above $r=0.7$. Furthermore, a total of 2 factors with a characteristic value that was larger than 1 were extracted through principle component analysis, whose cumulative contribution rate reached $80.241 \%$. Principle Component 1 represented factors of lumbar anteversion, which was related to the ratio of anterior/posterior waist dart's volume; and Principle Component 2 represented the lateral lumbar shape characteristics affecting the total waist dart's volume.
\end{abstract}

\section{Introduction}

Fitness is a core evaluating indicator of the quality of garment customisation ${ }^{[1]}$. In the field of bottom patternmaking for individuals, it is effective if the ratio of waist dart's volumes is matched with different lumbar shapes, so as to improve the fitness of bottoms. However, the waist-hip difference is a standard and the only parameter related to the total waist dart's volume for clothing mass production without taking the features of individual lumbar shapes into account.

There are many theoretical researches on parametric descriptions of somatotype differences and human body shape subdivisions [2] were oriented to garment customisations, according to statistical analyses based on multiple body shape variables collected via threedimensional human body scanning method. Meanwhile, it is a widespread and potential approach that was applied in the field of basic researches, such as body type classification and garment customisation, etc.

There are some researches related to pattern making, such as parametrises of pants back piece corresponding to 4 types of gluteal groove morphologies by draping ${ }^{[3]}$; the clustering of upper crotch length of man's trousers based on linear regression relationships with lower body height variables ${ }^{[4]}$ as well as the linear regression model of garment shoulder angle based on BMI and shoulder angle ${ }^{[5]}$, through which the relationships between human body parameters and garment patterns were discussed based on statistical analyses considering differences in human body shapes.
For the dart's volume determination, studies on the waist-darts volume defined by standard young women mannequins ${ }^{[6]}$ represented the average ratio of waistdarts, which was inadaptable to performing differences in human body shapes. Mathematical predicting models of breast and the back dart volumes were respectively established with characteristic variables of breast and acromion based on geometric features of chest and back of female bodies ${ }^{[7]}$, and discussions on body shape characteristics were limited to specified geometric parameters that showed standardized human body shapes. Otherwise, Keiko Hiraragi ${ }^{[8]}$ analysed relationships between morphological characteristics of female upper bodies and the ratio of each waist-dart's volume in a trunk basic pattern according to classifications of upper body shapes. This study provided theoretical clues for darts volume assignments related to garment pattern-making, which took differences in human body shapes into consideration.

Therefore, the purpose of this study is to extract lumbar shape factors influencing the ratio of waist dart's volumes based on characteristic analyses about lumbar shapes of young females. In this paper, lumbar shapes and dart's volumes measured through three-dimensional human body cross sections are main data sources for lumbar shape feature analyses.

\section{Method of Data Acquisition of Lumbar}


The subjects 72 young women aged 18 to 25 years old, were asked to wear light-coloured fitting shorts and vests.

\subsection{Measurement Points of Lumbar}

According to the definition of human body surface measurement point ${ }^{[9]}$, there are 4 measurement points manually confirmed on lumbar surface, including lateral waist point, abdomen projected point, buttock projected point and maximum thigh point, all of which were marked with labels initially.

\subsection{Method of Three-dimensional Human Body Scanning and Overlapping of Cross-sections}

Body surfaces were scanned via an OKIO-Body Scan Tian Yuan human three-dimensional scanner. During the scanning, arms of subjects were spread out for about $30^{\circ}$, kept upright and looking ahead.

Characteristic cross-sections of lumbar belonging to aforementioned lumbar measurement points were selected and input into AutoCAD; then, these characteristic cross-sections were overlapped as a measuring graphics.



The measuring method of overlapping graphic of lumbar cross-sections is shown in Figure 1, and measurement procedures are as follows: 1) Via the waist dart measuring method [9], encircling lines of characteristic cross-sections of lumbar are drawn through connecting the measurement feature points; 2) The $\mathrm{x}$-axis $(1 / 2$ point of the distance between central point ' $\mathrm{f}$ ' of the waist thickness and central point ' $\mathrm{e}$ ' of the outer circumferential thickness) and the y-axis ( $1 / 2$ point of the waist breadth) intersect at point ' $\mathrm{o}$ ', then take the length of $1 / 2$ waist thickness ( $\left.c^{\prime} \mathrm{o}^{\prime}\right)$ on $y$-axis to determine the focal point ' $\mathrm{o}$ ' ; 3) Take the focal point ' $\mathrm{o}$ " as central point, draw the rays at an interval of $30^{\circ}$ as waist dart lines and the midlines between each two adjacent waist dart lines (dotted line, II-V).

\subsection{Measurement Items of Overlapping Graphic of Lumbar Cross-sections}

A total of 27 items were measured on the overlapping graphic of lumbar cross-sections, including 21 for the measurement of lumbar shapes and 6 for that of waistdarts.

Measurement items of lumbar shapes include circumferences, widths and thicknesses of lumbar, and calculation items include waist flattening rate, etc.

A difference between encircling and waist lines is the total waist dart volume; and a difference among midlines of each two adjacent waist-dart lines is each waist-dart's volume. A part of definitions of lumbar shape measurement items and waist-dart's ratios are shown in Table 1.

Figure 1. Waist overlapping sections diagram.

Table 1. Measurement items in the graphic of partial waist overlapping sections.

\begin{tabular}{cll}
\hline Ordina & Measuring project & \multicolumn{1}{c}{ Definition } \\
\hline 1 & $\begin{array}{l}\text { Buttock projected } \\
\text { volume }(\mathrm{ab})\end{array}$ & The distance between the encircling and the WL line at the front center. \\
2 & $\begin{array}{l}\text { Abdomen projected } \\
\text { volume }(\mathrm{cd})\end{array}$ & $\begin{array}{l}\text { The distance between the outer perimeter and the WL line at the back } \\
\text { center. }\end{array}$ \\
3 & $\begin{array}{l}\text { Lumbar anteverted } \\
\text { amount (ytq) }\end{array}$ & $\begin{array}{l}\text { The distance between the outer perimeter thick coronal line 'e' and the } \\
\text { WL thick coronal line ' } \mathrm{f} \text { '. }\end{array}$ \\
4 & $\begin{array}{l}\text { Lateral lumbar } \\
\text { bending level (ytc) }\end{array}$ & $\begin{array}{l}\text { The distance between outer perimeter line of lateral lumbar line and } \\
\text { WL line. }\end{array}$ \\
6 & $\begin{array}{l}\text { Lumbar anteversion } \\
\text { angle }(\beta) \\
\text { Total waist dart's } \\
\text { volume }(\mathrm{s})\end{array}$ & The angle of a right triangle with 'ytq' and 'ytc' as right-angled edges. \\
\end{tabular}


$7 \quad$ Dart $1(\%)$

$8 \quad$ Dart $2(\%)$

$9 \quad$ Dart $3(\%)$

$10 \quad$ Dart $4(\%)$

$11 \quad$ Dart $5(\%)$
The difference between the encircling line and the WL line between Front Center I and Dart Midline II, and its percentage to the total dart's volume.

The size difference between the outer perimeter and the WL perimeter between Dart Midline II and Dart Midline III accounting for the percentage of the total dart's volume.

The size difference between the outer perimeter and the WL perimeter between Dart Midline III and Dart Midline IV accounting for the percentage of the total dart's volume.

The size difference between the outer perimeter and the WL perimeter between Dart Midline IV and Dart Midline V accounting for the percentage of the total dart's volume.

The size difference between the outer perimeter and the WL perimeter between Dart Midline V and Posterior Central VI accounting for the percentage of the total dart's volume.

\section{Basic Statistics of Lumbar Shape}

According to results of Kolmogorov-Smirnov test through SPSS statistics 23, all lumbar feature variables follow a normal distribution.
Taking A-type upper body size in GB/T+1335.22008--"standard sizing for garment-women" as a test value, one-sample t-tests were done, whose results are shown in Table 2. The average waist circumference of subjects taken in this study was significantly different from the national standard of A-type, which showed the basic characteristics of subjects taken in this study.

Tab 2. Results of single-sample t-tests at the control site compared with national A-type women. $n=30$

\begin{tabular}{cccccc}
\hline $\begin{array}{c}\text { Control } \\
\text { variable }\end{array}$ & $\begin{array}{c}\text { Average } \\
\text { value }(\mathrm{cm})\end{array}$ & $\begin{array}{c}\text { Standard } \\
\text { deviation }\end{array}$ & $\begin{array}{c}\text { Inspection value }(\mathrm{cm}) \\
160 / 84 \mathrm{~A}\end{array}$ & $t$ & Significance \\
\hline Height & 160.00 & 4.70 & 160.00 & -0.008 & N.S. $^{\text {a }}$ \\
WL & 66.42 & 4.20 & 68.00 & -2.067 & $*$ b $^{\text {a }}$ \\
HL & 90.84 & 4.88 & 90.00 & 0.948 & N.S. $^{\text {a }}$ \\
\hline
\end{tabular}

${ }^{\mathrm{a}} \mathrm{p}>0.05$ : No significant difference;

${ }^{\mathrm{b}} 0.01<\mathrm{p}<0.05$ : difference;

${ }^{\mathrm{c}} \mathrm{p}<0.01$ : Significant difference.

4 Correlative Relationships between Waist-darts Volume and Lumbar Shape Features
The results of correlation analyses between waist-darts and the measurement of lumbar morphological features on overlapped diagrams are shown in Table 3. Abdomen projected volumes (cd) respectively showed a highly significant correlation over $\mathrm{r}=0.8$ with the waist darts.

Tab 3. Results of correlation analyses between waist shape variables and the allocation rate of dart's volume.

\begin{tabular}{cccccc}
\hline Waist dart & $\begin{array}{c}\text { Lumbar } \\
\text { anteversion angle } \\
(\beta)\end{array}$ & $\begin{array}{c}\text { Lumbar } \\
\text { anteved amount } \\
(\mathrm{ytq})\end{array}$ & $\begin{array}{c}\text { Buttock projected } \\
\text { volume (ab) }\end{array}$ & $\begin{array}{c}\text { Abdomen } \\
\text { projected volume } \\
(\mathrm{cd})\end{array}$ & $\begin{array}{c}\text { Lateral lumbar } \\
\text { bending level (ytc) }\end{array}$ \\
\hline Total waist dart's volume & 0.031 & 0.223 & $.389^{*}$ & 0.151 & $.830^{* *}$ \\
Dart 1 (\%) & $-.917^{* *}$ & $-.910^{* *}$ & $-.780^{* *}$ & $.865^{* *}$ & 0.15 \\
Dart 2 (\%) & $-.841^{* *}$ & $-.814^{* *}$ & $-.863^{* *}$ & $.494^{* *}$ & 0.238 \\
Dart 3 (\%) & $-.367^{*}$ & -0.312 & $-.509^{* *}$ & -0.092 & 0.244 \\
Dart 4 (\%) & $.796^{* *}$ & $.813^{* *}$ & $.774^{* *}$ & $-.625^{* *}$ & 0.015 \\
Dart 5 (\%) & $.949^{* *}$ & $.911^{* *}$ & $.896^{* *}$ & $-.685^{* *}$ & -0.302 \\
\hline
\end{tabular}

\footnotetext{
${ }^{* *}$ At level 0.01 (two-tailed), the correlation is significant;
}

${ }^{*}$ At level 0.05 (two-tailed), the correlation is significant. 
A significant correlation of $\mathrm{r}=0.815$ was shown between the total waist-dart's volume and the lateral lumbar bending level (ytc), which indicated a close relationship between the side waist shape and the total waist dart's volume.

Morphological variables of lumbar anteversion angle $(\beta)$, lumbar anteversion amount (ytq), lateral lumbar bending level (ytc) and buttock projected volume (ab) were negatively correlated with the ratio of front waist dart's volumes over $\mathrm{r}=0.7$ (Dart 1\%, Dart $2 \%$ ), which were positively correlated to the ratio of back waist-dart's volumes (Dart 4, Dart 5) respectively. It is shown that the ratio of front waist-dart's volumes decreased with increases in the degree of lumbar anteversions, and the ratio of back waist-dart's volumes increased with increases in the degree of lumbar anteversions.

\section{Extraction of Lumbar Shape Feature Factors Affecting the Ratio of Waist Dart's Volumes}

Based on the relationship shown between the ratio of waist-dart's volume and the lumbar morphological feature variables, principle component analyses were carried out to extract lumbar shape feature factors affecting the ratio of waist-dart's volumes. 11 variables featured lumbar shapes were selected after eliminating variables uncorrelated with waist-dart's volumes and verified non-collinear. Results of principle component analyses through maximum variance method are shown in Table 4.

A total of 2 factors with a characteristic root that was larger than 1 were extracted, whose cumulative contribution rate reached $80.241 \%$. In Principle Component 1, ratios of front and back waist dart's volumes (Dart 1, Dart 2, Dart 4 and Dart 5) and characteristic variables $(\beta$, ytq, and ab) showed a high loading coefficient that was above 0.7 , thus, this principle component could be interpreted as a lumbar anteversion factor; in Principle Component 2, the total waist-dart's volume and the lateral lumbar bending level (ytc) showed a high loading coefficient that was above 0.8 , which was interpreted as a lateral lumbar shape factor.

In summary, two factors influencing the ratio of waist-dart's volumes were extracted, of which Feature Factor 1 affected the ratio of front and back waist-dart's volumes, and Feature Factor 2 affected the total waistdart's volume.

Tab 4. Results of factor analyses.

\begin{tabular}{ccc}
\hline Variable & Factor \\
\hline Lumbar anteverted amount (ytq) & 1 & 2 \\
Lumbar anteversion angle $(\beta)$ & 0.972 & 0.099 \\
Dart 5(\%) & 0.971 & -0.081 \\
Dart 1 (\%) & 0.961 & -0.124 \\
Duttock projected volume (ab) & -0.932 & 0.137 \\
Dart 2 (\%) & 0.901 & 0.257 \\
Dart 4 (\%) & -0.888 & 0.023 \\
Abdomen projected volume (cd) & 0.853 & 0.133 \\
Lateral lumbar bending level (ytc) & -0.755 & 0.239 \\
Total waist-dart's volume & -0.017 & 0.888 \\
Wide outer perimeter & 0.264 & 0.885 \\
Characteristic root & -0.222 & 0.602 \\
Variance contribution rate $(\%)$ & 6.700 & 2.127 \\
Cumulative contribution rate $(\%)$ & 60.906 & 19.335 \\
\hline
\end{tabular}

\section{Conclusion}

Waist morphological features that affected the ratios of waist-dart's volumes of young women were extracted in this study. Main conclusions are as follows:

1)In the results of correlation analyses, the total waist-dart's volume significantly showed a correlative relationship with the lateral lumbar bending level (ytc), and the ratio of waist-dart's volumes was significantly correlated with variables representing the degree of lumbar anteversions.

2)Two principle component factors influencing the ratios of waist-dart's volumes were extracted through principle component analysis. Principle Component 1 was interpreted as a lumbar anteversion factor that affected the ratio of each waist-dart's volumes; and Principle Component 2 was interpreted as a lateral lumbar factor that affected the total waist-dart's volume.

\section{References}

1. Hwa K S and Susan P A 2012 Development of Automated Custom-Made Pants Driven by Body Shape J. Clothing and Textiles Research Journal. vol 30, no 4. 315-329

2. Hwa K S and Susan P A 2011 Categorization of lower body shapes for adult females based on multiple view analysis $J$. Textile Research Journal. vol 81, no 9. 315-329

3. Xia Y, Shi X Q and Wang H F 2015 Analysis on young female's hip convex curve and trousers' 
back part structure J. Journal of Textile Research. vol 36, no 11. 115-120

4. Wang Y B, Wang H F and Ke Y 2018 Influential factors of upper crotch length in young male's pants J. Journal of Textile Research. vol 39, no 9. 115-119

5. Li X H, Zhang S Y, Zhao C T and Bian F 2018 Relationship between garment shoulder angle and characteristics of human body J. Journal of Textile Research. vol 39, no 11. 111-115

6. Zhao X G, Pan L, Wang J, Yu Z J, Yao T, Zhou B X and Wang X N 2015 Method of chest-waist difference distribution for clothing prototype of young women in northeast China J. Journal of Dalian Polytechnic University. vol 34, no 5. 370373

7. Jiang L J and Wang W L 2014 Elationship between Female Bust and Back Characteristics and the Individual Prototype Darts J. Journal of Dong hu a University (Natural Scence). vol 40, no 5. 571-574

8. Keiko H 2013 A Study on the Allocation Ratio of Women's Upper Half Body Prototype Waist Darts on Different Body Types-Body Classification Using a Polymerization Horizontal Section Figure- $J$. Bunka Gakuen University. vol 44. 1-11

9. Milyoshi M 2000 The Theory of Fashion Modeling, 1st ed (Beijin: China Textile \& Apparel Press) $p$ 116,178 